\title{
EXTREME INVARIANT MEANS WITHOUT MINIMAL SUPPORT $\left({ }^{1}\right)$
}

BY

\section{LONNIE FAIRCHILD}

\begin{abstract}
Let $S$ be a left amenable semigroup. We show that if $S$ has a subset satisfying a certain condition, then there is an extreme left invariant mean on $S$ whose support is not a minimal closed invariant subset of $\beta S$. Then we show that all infinite solvable groups and countably infinite locally finite groups have such subsets.
\end{abstract}

1. Introduction. If $S$ is a left amenable semigroup with the discrete topology and $\beta S$ its Stone-Cech compactification, then $S$ acts on $\beta S$ by the continuous extension to $\beta S$ of left translations by elements of $S$. There is a one-to-one correspondence between the ergodic measures on $\beta S$ and the extreme points of the set of left invariant means on $m(S)$. It is easy to show that every minimal closed invariant subset of $\beta S$ is the support of at least one ergodic measure. Wilde, in [8], gives a condition for the support of every ergodic measure on $\beta S$ to be a minimal closed invariant set, and Chou has proved that there are ergodic measures on $\beta N$ without minimal support [1].

In $\$ 3$ we show that a necessary and sufficient condition for the existence of such nonminimally supported ergodic measures is the following: $S$ has a subset $A$ such that $\phi\left(\chi_{A}\right)>0$ for some left invariant mean $\phi$ on $m(S)$, but $\psi\left(\chi_{K-1}\right)<1$ for every left invariant mean $\psi$ and every finite subset $K$ of $S$. In $\$ 4$ we prove that every countably infinite locally finite group contains such a subset and that every infinite solvable group has a subset $A$ with the following stronger property: $\phi\left(\chi_{A}\right)$ $>0$ for some left invariant mean $\phi$ on $m(S)$, and for each positive integer $k$ there is a finite set $S_{k} \subset S$ such that $S_{k} s \not \subset K^{-1} A$ for every $s \in \mathrm{S}$ and every subset $K$ of $S$ with $|K| \leq k$.

2. Preliminaries. We will follow Day [2] for notation and terminology on invariant means. Let $S$ be a semigroup, $m(S)$ the Banach space of bounded real functions on $S$ with the sup norm, $m(S)^{*}$ the dual of $m(S)$, and $l_{1}(S)$ the space of real functions $\theta$ on $S$ such that $\|\theta\|_{1}=\Sigma_{s \in S}|\theta(s)|$ is finite. Let $Q$ denote the isometry from $l_{1}(S)$ into $m(S)^{*}$ given by $Q \theta(f)=\Sigma_{s \in S} f(s) \theta(s)$ for $\theta \in l_{1}(S)$ and $f \in m(S)$. If we identify each element $s$ of $S$ with the characteristic function $\chi_{\{s\}}$ in $l_{1}(S)$, we get $Q s(f)=f(s)$ for all $f \in m(S)^{*}$. For $s$ in $S$, the operator $l_{s}$ from $m(S)$ into $m(S)$ is

Presented to the Society, April 4, 1971; received by the editors June 30, 1971.

AMS (MOS) subject classifications (1970). Primary 43 A07.

Key words and phrases. Amenable semigroup, extreme point, Stone-Čech compactification, minimal invariant set.

( $\left.{ }^{1}\right)$ This paper contains results from the author's $\mathrm{Ph}$. D. thesis (University of Illinois, June 1970). The author wishes to thank her advisor, Professor M. M. Day, for his help. 
defined by $l_{s} f(t)=f(s t)$ for all $t$ in $S$ and $f$ in $m(S)$. Let $l_{s}^{*}$ be the adjoint of $l_{s}$. $M(S)$ will denote the set of means on $m(S)$, and $M l(S)$ the set of left invariant means. We will regard $\beta S$ as the set of multiplicative means with the $w^{*}$ topology. If $U$ is a subset of $\beta S, U^{-}$will denote the $w^{*}$ closure of $U$. If $A \subset S$, then $(Q A)^{-}$ is open in $\beta S$ and all the open and closed subsets of $\beta S$ are of this form. $\beta S$ is extremally disconnected and the open and closed sets form a base for the topology. For other properties of $\beta S$, see Gillman and Jerison [3].

The Arens multiplication $\odot$ on $m(S)^{*}$ is defined as follows: If $\mu, \nu \in m(S)^{*}$ and $f \in m(S)$, let $\mu \odot \nu(f)=\mu(\nu \odot f)$ where $\nu \odot f(s)=\nu\left(l_{s} f\right)$ for all $s \in S$. This multiplication makes $m(S)^{*}$ into a Banach algebra. We will use the following properties of $\odot$ (see Day [2]):

(1) $M(S)$ and $\beta S$ are each closed under $\odot$. (2) If $s \in S$ and $\mu \in m(S)^{*}$, then $Q s \odot \mu=l_{s}^{*} \mu$. (3) $Q(s t)=Q s \odot Q t$ for all $s, t \in S$. (4) If $\nu \in m(S)^{*}$ is fixed, then the mapping $\mu \rightarrow \mu \odot \nu$ is $w^{*}-w^{*}$ continuous. If $\theta \in l_{1}(S)$ is fixed, the mapping $\mu \rightarrow$ $Q \theta \odot \mu$ is $w^{*}-w^{*}$ continuous. (5) If $\mu \in M l(S)$ and $\nu \in M(S)$ then $\mu \odot \nu \in M l(S)$ and $\nu \odot \mu=\mu$.

Let $C(\beta S)$ be the space of real-valued continuous functions on $\beta S$. Let $T$ be the isometric algebra isomorphism $C(\beta S)$ onto $m(S)$ given by $T f(s)=f(Q s)$ for all $s \in S$ and $f \in m(S)$. Define $P$ from $m(S)^{*}$ onto $C(\beta S)^{*}$ to be the adjoint of $T$. By the Riesz representation theorem we can regard $C(\beta S)^{*}$ as the space of regular Borel measures on $\beta S$.

If $K \subset S$ and $U \subset \beta S$, let $Q K^{-1} \odot U=\{\mu \in \beta S: Q s \odot \mu \in U$, for some $s \in K\}$. If $A \subset S$, let $K^{-1} A=\{t \in S: s t \in A$ for some $s \in K\}$. If $\mu \in m(S)^{*}$, then $\mu \in M l(S)$ iff $P \mu$ is a probability measure on $\beta S$ such that $P \mu\left(Q s^{-1} \odot U\right)=P \mu(U)$ for each $s \in S$ and each Baire subset $U$ of $\beta S$. $P \mu$ is called invariant if $\mu$ is left invariant. If $S$ is an infinite right cancellation semigroup and $\mu \in M l(S)$ then $P \mu(Q S)=0$.

An invariant probability measure $\lambda$ on $\beta S$ is called ergodic if $\lambda(A)=0$ or $\lambda(A)$ $=1^{\circ}$ for all Baire sets $A$ which satisfy $\lambda\left(A \Delta\left(Q s^{-1} \odot A\right)\right)=0$ for each $s \in S$. A left invariant mean $\mu$ on $m(S)$ is an extreme point of the convex set $M l(S)$ iff $P \mu$ is ergodic. (See Phelps [7, p. 81].)

A nonempty subset $U$ of $\beta S$ is said to be invariant if $Q s \odot U \subset U$ for all $s \in S$. In this case we have $\omega \odot U \subset U$ for all $\omega \in \beta S$. $U$ is called minimal if it is closed and invariant and minimal with respect to this property. If $\omega \in \beta S$, let $o(\omega)=$ $\{Q s \odot \omega: s \in S\}$. Then $o(\omega)^{-}$is a closed invariant set.

If $S$ is left amenable and $F$ is a closed invariant set, then for each $\omega \in F$ and $\mu \in M l(S), P(\mu \odot \omega)$ is an invariant measure with support contained in $F$. The following proposition was proved by Wilde and Witz [9, p. 583] for the case where $S$ has two-sided cancellation.

2.1 Proposition. If $S$ is a semigroup and $\mu \in M l(S)$ then the support of $P \mu$ is an invariant set. 
Proof. Suppose $\omega \in \operatorname{supp} P \mu$ and $s \in S$. Let $Q A^{-}$be a basic open neighborhood of $Q s \odot \omega$. Then $Q s^{-1} \odot Q A^{-}$is open and contains $\omega$. Thus, $0<P \mu\left(Q s^{-1} \odot Q A^{-}\right)$ $=P \mu\left(Q A^{-}\right)$. Since $Q A^{-}$was arbitrary, we get $Q s \odot \omega \in$ supp $P \mu$.

We say that an element $\omega$ of $\beta S$ is a left almost periodic point provided that for every neighborhood $U$ of $\omega$ there is a subset $A$ of $S$ satisfying: (1) there exists a finite subset $K$ of $S$ such that $S=K^{-1} A$, and (2) $Q A \odot \omega \subset U$.

3. Minimal sets and supports of invariant measures. In this section we consider the relations among the set $A^{S}$ of almost periodic points in $\beta S$, the set $B^{S}$ of elements of $\beta S$ which belong to some minimal set, and the set $K^{S}$ of points which are in the support of some invariant measure. We prove that $A^{S}=B^{S}$ and $A^{S} \subset K^{S}$ for any left amenable semigroup $S$. The main result is Proposition 3.4 in which we

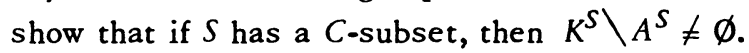

From now on $S$ is always assumed to be a left amenable semigroup. It is proved in Gottschalk and Hedlund [4, p. 32] that if $S$ is a group then $A^{S} \subset B^{S}$. We extend this by the following proposition.

3.1 Proposition. If $S$ is any left amenable semigroup, then $A^{S}=B^{S}$.

Proof. First, let $T$ be a minimal subset of $\beta S$ and assume $\omega \in T$. Since $T$ is minimal, $T=o(\omega)^{-}$. Let $U$ be an open neighborhood of $\omega$ and suppose there exists an element $\nu$ in $T \backslash\left(Q S^{-1} \odot U\right)$. If $s \in S$, then $Q s \odot \nu$ is in $T$ since $T$ is invariant. If $Q s \odot \nu$ is in $Q S^{-1} \odot U$, there exists $t \in S$ such that $Q(t s) \odot \nu=Q t \odot Q s \odot \nu \in U$. Then we get $\nu \in Q(t s)^{-1} \odot U$, which contradicts our assumption that $\nu$ is not in $Q S^{-1} \odot U$. Hence we must have $Q s \odot \nu \in T \backslash\left(Q S^{-1} \odot U\right)$, so $T \backslash\left(Q S^{-1} \odot U\right)$ is a closed invariant set. Since $T$ is minimal, this implies that $T \cap\left(Q S^{-1} \odot U\right)=\emptyset$. But then $\omega$ is not in $Q S^{-1} \odot U$ and $o(\omega) \cap U=\emptyset$. This is a contradiction since $U$ is open and $\omega \in T=o(\omega)^{-}$. Therefore, $T \backslash\left(Q S^{-1} \odot U\right)=\varnothing$ and $T \subset Q S^{-1} \odot U$. Since $T$ is compact, there is a finite subset $K$ of $S$ such that $T \subset Q K^{-1} \odot U$. Let $A=\{s \in S: Q s \odot \omega \in U\}$. If $s \in S$, there is a $k \in K$ such that $Q(k s) \odot \omega=Q k \odot Q s \odot \omega$ $\in U$, so $k s \in A$. Hence $S=K^{-1} A$ and $\omega \in A^{S}$.

Now, assume $\omega \in A^{S}$ and let $T$ be a minimal subset of $o(\omega)^{-}$. Suppose $\omega$ is not in $T$. Let $U$ be an open neighborhood of $\omega$ such that $U^{-} \cap T=\varnothing$. S has a subset $A$ and a finite subset $K$ such that $Q A \odot \omega \subset U$ and $S=K^{-1} A$. Since $k \in K$, $s \in S$, and $k s \in A$ implies that $Q s \odot \omega \in Q k^{-1} \odot Q A \odot \omega \subset Q k^{-1} \odot U$, we have $o(\omega) \subset$ $Q K^{-1} \odot U$. Hence $o(\omega)^{-} \subset\left(Q K^{-1} \odot U\right)^{-} \subset Q K^{-1} \odot U^{-}$. If $k \in K$ and $\left(Q k^{-1} \odot U^{-}\right) \cap$ $T \neq \varnothing$, for some $\mu \in \beta S$ we would have $Q k \odot \mu \in U^{-} \cap(Q k \odot T) \subset U^{-} \cap T=\varnothing$, which is impossible. This gives $T \subset\left(\circ(\omega)^{-} \cap T\right) \subset\left(Q K^{-1} \odot U^{-}\right) \cap T=\emptyset$, which contradicts $T \neq \varnothing$. Therefore, $\omega \in T$.

3.2 Corollary。 $A^{S} \subset K^{S}$.

Proof. If $T$ is minimal and contains the support of $P \mu$, then $T=\operatorname{supp}(P \mu)$. 
If $A \subset S$, we define $\bar{d}(A)$ to be $\sup \left\{\phi\left(\chi_{A}\right): \phi \in M l(S)\right\}$. Mitchell [6, p. 253] proved that for each $f$ in $m(S)$, the set $\{\phi(f): \phi \in M l(S)\}$ is a closed interval. Hence, $\bar{d}(A)=1$ iff $\phi\left(\chi_{A}\right)=1$ for some $\phi \in M l(S)$.

If $A \subset S$, we call $A$ a $C$-subset of $S$ provided that

(a) $\bar{d}(A)>0$, and

(b) $\bar{d}\left(K^{-1} A\right)<1$ for every finite subset $K$ of $S$.

3.3 Lemma. If $S$ is a semigroup, $A \subset S$, and $s \in S$, then $Q s^{-1} \odot Q A^{-}=$ $\left(Q s^{-1} \odot Q A\right)^{-}=Q\left(s^{-1} A\right)^{-}$.

Proof. Clearly, $Q\left(s^{-1} A\right)^{-} \subset\left(Q s^{-1} \odot Q A\right)^{-} \subset Q s^{-1} \odot Q A^{-}$. Suppose $\omega \in Q s^{-1} \odot$ $Q A^{-}$. Then $Q s \odot \omega \in Q A^{-}$. Let $\left(Q t_{\alpha}\right)$ be a net in $Q S$ which converges to $\omega$. Then $\lim Q\left(s t_{\alpha}\right)=\lim \left(Q s \odot Q t_{\alpha}\right)=Q s \odot \omega \in Q A^{-}$. Since $Q A^{-}$is open, there exists $\alpha_{0}$ such that $\alpha>\alpha_{0}$ implies $Q\left(s t_{\alpha}\right) \in Q A^{-}$. But $Q S \cap Q A^{-}=Q A$, so $s t_{\alpha} \in A$ for $\alpha>\alpha_{0}$ and thus $t_{\alpha} \in s^{-1} A$. Hence $\omega=\lim Q t_{\alpha} \in Q\left(s^{-1} A\right)^{-}$, which shows that $Q s^{-1} \odot$ $Q A^{-}=Q\left(s^{-1} A\right)^{-}$.

3.4 Proposition. Suppose $S$ contains a C-subset A. Then $Q A^{-} \cap\left(A^{S}\right)^{-}=\varnothing$ and $K^{S} \cap Q A^{-} \neq \varnothing$ (and thus $A^{S} \varsubsetneqq K^{S}$ ).

Proof. Assume there exists $\omega \in Q A^{-} \cap A^{S}$. Then, since $2 A^{-}$is open, $S$ has a subset $B$ and a finite subset $K$ such that $S=K^{-1} B$ and $Q B \odot \omega \subset Q A^{-}$. Thus, $o(\omega)=Q\left(K^{-1} B\right) \odot \omega \subset Q K^{-1} \odot Q B \odot \omega \subset Q K^{-1} \odot Q A^{-}=Q\left(K^{-1} A\right)^{-}$, by Lemma 3.3. Therefore, $o(\omega)^{-} \subset Q\left(K^{-1} A\right)^{-}$. Since $o(\omega)^{-}$is a closed invariant set, there exists $\phi \in M l(S)$ such that $\operatorname{supp}(P \phi) \subset Q\left(K^{-1} A\right)^{-}$. Then $\phi\left(\chi_{K-1 A}\right)=1$, which contradicts the definition of $C$-subset. Thus $Q A^{-} \cap A^{S}=\emptyset$. Since $Q A^{-}$is open, $Q A^{-} \cap\left(A^{S}\right)^{-}=$ $\varnothing$. But, since $\bar{d}(A)>0, Q A^{-} \cap K^{S} \neq \varnothing$.

3.5 Corollary. Suppose $S$ has a $C$-subset $A$. Then there is an extreme point $\phi$ of $M l(S)$ such that the support of $P \phi$ is not a minimal set.

Proof. There exists $\psi \in M l(S)$ with $\psi\left(\chi_{A}\right)>0$, so there is an extreme point $\phi$ of $M l(S)$ with $\phi\left(\chi_{A}\right)>0$. Then $Q A^{-} \cap \operatorname{supp}(P \phi) \neq \emptyset$. But $Q A^{-} \cap A^{S}=\varnothing$, so by Proposition 3.1, $\operatorname{supp}(P \phi)$ is not a minimal set.

Chou implicitly proves 3.4 and 3.5 in [1] for the case where $S=N$. Since $Q A^{-} \cap\left(A^{S}\right)^{-}=\emptyset$ in Corollary 3.5, we have actually shown that the support of $P \phi$ properly contains the closure of the set $U\{T \subset \operatorname{supp}(P \phi): T$ is a minimal set $\}$. Thus $\phi$ is not in the $w^{*}$ closed convex hull of the set $\{\mu \in M l(S): \operatorname{supp}(P \mu)$ is a minimal set\}.

3.6 Proposition. Suppose $S$ does not bave any C-subsets. Then $K^{S} \subset\left(A^{S}\right)^{-}$.

Proof. Let $\omega$ be in $K^{S}$ and let $U$ be an open neighborhood of $\omega$. There is a subset $A$ of $S$ such that $\omega \in Q A^{-} \subset U$. There is a left invariant mean $\phi$ with $\phi\left(\chi_{A}\right)>0$. Since $A$ is not a $C$-subset of $S$ there is a finite subset $K$ of $S$ such 
that $\bar{d}\left(K^{-1} A\right)=1$. Thus there exists $\psi \in M l(S)$ such that $\operatorname{supp}(P \psi) \subset Q\left(K^{-1} A\right)^{-}$. Hence there is a minimal set $T \subset Q\left(K^{-1} A\right)^{-} \subset Q K^{-1} \odot Q A^{-}$. Choose $\omega_{0} \in T$ and $k \in K$ so that $Q K \odot \omega_{0} \in Q A^{-} \subset U$. Then $Q k \odot \omega_{0} \in T \cap U$, so $U \cap A^{S} \neq \varnothing$ by Proposition 3.1. Since $U$ was arbitrary, we get $\omega \in A^{S}$.

4. Semigroups with $C$-subsets and $C^{\prime}$-subsets. In this section we prove the existence of $C$-subsets for certain semigroups.

$Z$ will denote the additive group of integers, and $N$ the positive integers. If $x, y \in Z$, let $[x, y]=\{a \in Z: x \leq a \leq y\}$. If $k \in N$, a $k$-chain is a subset $A$ of $Z$ such that $|c-d| \leq k$ whenever $c$ and $d$ are two consecutive elements of $A$. A $k$-chain $A$ has length $n$ if $|A|=n$. If $B$ is a set, $|B|$ denotes the cardinality of $B$.

A subset $A$ of a semigroup $S$ is called left thick if for every finite subset $K$ of $S$, there exists $s_{K} \in S$ such that $K s_{K} \subset A$. We need the following theorem.

4.1 Theorem (Mitchell [6, p. 257]). If $S$ is a left amenable semigroup and $A \subset$ $S$, then $\bar{d}(A)=1$ iff $A$ is left thick.

A subset $A$ of $S$ is said to be a $C^{\prime}$-subset of $S$ if

(1) $\bar{d}(A) \geq 0$, and

(2) for each $k \in N$, there exists a finite subset $S_{k}$ of $S$ such that for all $s^{\prime} \epsilon$ $S$ and $K \subset S$ with $|K| \leq k$ we have $S_{k} s^{\prime} \not \subset K^{-1} A$.

Condition (2) implies that for each finite set $K, K^{-1} A$ is not left thick, so by Theorem 4.1, every $C^{\prime}$-subset of $S$ is a $C$-subset.

Chou [1] has constructed a $C$-subset of $N$ (or $Z$ ). We will generalize this construction to show that $Z$ (or $N$ ) has a large number of $C^{\prime}$-subsets.

4.3 Theorem. If $A$ is a left thick subset of $Z$ (or $N$ ), then A contains a $C^{\prime}$-subset $D$ of $Z$ (or $N)$.

Proof. We will prove the proposition for $Z$. Either $A \cap N$ or $A \cap(-N)$ is left thick. We will assume $A \cap N$ is left thick; a symmetrical argument works in the other case.

By the definition of left thickness, there exists $b_{1}$ in $A$ such that $b_{2}=b_{1}+$ $1 \in A$. Let $B_{1}=\left\{b_{1}, b_{2}\right\}$. Assume $B_{1}, B_{2}, \cdots, B_{k}$ and $b_{1}, \cdots, b_{p}$, where $p=$ $\Sigma_{i=1}^{k} 2^{i}$, are defined. There exists $b_{p+1} \in A$ such that $b_{p+1}>b_{p}$ and $B_{k+1}=$ $\left[b_{p+1}, b_{p+1}+2^{k-1}-1\right] \subset A$. Let $b_{p+j+1}=b_{p+1}+j$ for $j=0,1, \cdots, 2^{k+1}-1$. Then $B=\bigcup\left\{B_{k}: k \in N\right\}$ is a left thick subset of $A$.

Let $A_{1}=\left\{b_{1}, b_{2}, b_{3}\right\}$. Assume we have constructed $A_{1}, \cdots, A_{n}$. Let $a(n)=$ $\left|A_{n}\right|$ and let $d(n)=\left|\left\{b_{i} \in B: b_{i} \leq \sup A_{n}, b_{i} \in A_{n}\right\}\right|$. Define $A_{n+1}=A_{n} \cup$ $\left\{b_{a(n)+d(n)+n+j}: b_{j} \in A_{n}\right\} \cup\left\{b_{2 a(n)}+2 d(n)+2 n+j: b_{j} \in A_{n}\right\}$. Let $D=\bigcup\left\{A_{i}: i \in N\right\}$. If we let $J_{n}=\left\{i: b_{i} \in A_{n}\right\}$ for each $n$ in $N$, then we get $J_{n+1}=J_{n} \cup\left(J_{n}+n+\right.$ $\left.\sup J_{n}\right) \cup\left(J_{n}+2 n+2 \sup J_{n}\right)$.

For $n$ in $N$, define $\phi_{n}$ in $M(Z)$ by $\phi_{n}(f)=(1 / n) \Sigma_{i=1}^{n} f\left(b_{i}\right)$ for all $f$ in $m(Z)$. 
We will show that the sequence $\left(\phi_{n}\right)$ is $w^{*}$ convergent to left invariance. For each $n$, let $k(n)$ be the integer which satisfies $b_{n} \in B_{k(n)}$. Then $n \geq 2^{k(n)}-1$, and

$$
\begin{aligned}
\mid \phi_{n}(f) & -\phi_{n}\left(l_{1} f\right)\left|=\frac{1}{n}\right| \sum_{i=1}^{n} f\left(b_{i}\right)-f\left(b_{i}+1\right) \mid \\
& =\frac{1}{n}\left|\sum_{j=1}^{k(n)} \sum_{\left\{i \leq n: b_{i} \in B_{j}\right\}} f\left(b_{i}\right)-f\left(b_{i}+1\right)\right| \\
& \leq 2\|f\| k(n) / n \leq 2\|f\| k(n) / 2^{k(n)-1} .
\end{aligned}
$$

Thus $\lim \left|\phi_{n}(f)-\phi_{n}\left(l_{1} f\right)\right|=0$. Let $\psi_{n}=\phi_{a(n)}+d(n)$ for each $n$ in $N$ and let $\psi$ be a $w^{*}$ limit point of the sequence $\left(\psi_{n}\right)$. Then $\psi \in M l(Z)$. We have

$$
\psi_{n}\left(\chi_{D}\right)=\left|D \cap\left\{b_{1}, b_{2}, \cdots, b_{a(n)+d(n)}\right\}\right| /(a(n)+d(n))=a(n) /(a(n)+d(n)) .
$$

We have $a(n+1)=3 a(n)$ for $n$ in $N$, and $a(1)=3$. Hence $a(n)=3^{n}$ for each $n$. Also $d(n+1)=3 d(n)+2 n$ for $n$ in $N$ and $d(1)=0$. Hence $d(n)=1-n+\left(3^{n}-3\right) / 2$. This gives $\psi_{n}\left(\chi_{D}\right)=3^{n} /\left(3^{n}+1-n+\left(3^{n}-3\right) / 2\right) \geq 2 / 3$, so $\bar{d}(D) \geq \psi\left(\chi_{D}\right) \geq 2 / 3$.

Note that if $k \in N$, every $k$-chain in $D$ has length $\leq a(k)$. Also, if $E$ is a $k$-chain of length $n$ then $(\sup E-\inf E) \leq(n-1) k$, and if $y-x \geq n k+k$ then $[x, y]$ contains an interval of the form $[p, p+k-1]$ which does not meet $E$. We will use the following lemma to show that $D$ satisfies condition (2) for a $C^{\prime}$-subset.

4.4 Lemma. Assume $k \in N$. Then for each $j \in N$ there exists $p_{k}(j) \in N$ such that, for any finite subset $K$ of $Z$ with $|K| \leq k$ and for any $z \in Z$, there exists $z_{0} \epsilon$ $\left[z, z+p_{k}(j)-j\right]$ with $\left[z_{0}, z_{0}+j-1\right] \cap(-K+D)=\emptyset$.

Proof. The proof is by induction on $k$. Take $p_{1}(j)=j a(j)+j$ for each $j$ in $N$. Since $D$ contains no $j$-chain of length $\geq a(j)$, neither does $-s+A_{0}$ for any $s$ in $Z$. Then, if $z \in Z$, the set $\left[z, z+p_{1}(j)-1\right] \cap\left(-s+A_{0}\right)$ is not a $j$-chain. Thus, there exists $z_{0} \in\left[z, z+p_{1}(j)-j\right]$ such that $\left[z_{0}, z_{0}+j-1\right] \cap\left(-s+A_{0}\right)=\varnothing$, so the $p_{1}(j)$ 's satisfy the lemma.

Now, assume there exist $p_{n}(j)$ 's satisfying the lemma. Let $p_{n+1}(j)=p_{n}\left(p_{1}(j)\right)$ for each $j \in N$. Suppose $K \subset Z$ and $1 \leq|K| \leq n+1$. We can write $K=K_{n} \cup\{a\}$ where $\left|K_{n}\right| \leq n$. Then $-K+D=\left(-K_{n}+D\right) \cup(-a+D)$. Suppose $z \in Z$. There exists $z_{0} \in\left[z, z+p_{n}\left(p_{1}(j)\right)-p_{1}(j)\right]$ such that $\left[z_{0}, z_{0}+p_{1}(j)-1\right] \cap\left(-K_{n}+A_{0}\right)=$ $\emptyset$. There exists $z_{0}^{\prime} \in\left[z_{0}, z_{0}+p_{1}(j)-j\right]$ such that $\left[z_{0}^{\prime}, z_{0}^{\prime}+j-1\right] \cap\left(-a+A_{0}\right)=$ $\emptyset$. Then $z_{0}^{\prime}$ is in $\left[z, z+p_{n-1}(j)-j\right]$ and $\left[z_{0}^{\prime}, z_{0}^{\prime}+j-1\right] \cap\left(-K_{n}+A_{0}\right)=\varnothing$. Hence, $\left[z_{0}^{\prime}, z_{0}^{\prime}+j-1\right] \cap\left(-K+A_{0}\right)=\emptyset$ and the $p_{n+1}(j)$ 's satisfy the lemma.

To complete the proof of Theorem 4.3, let $S_{k}=\left[0, p_{k}(1)-1\right]$ for each $k \in N$. Then if $s \in Z$, we have $S_{k}+s=\left[s, s+p_{k}(1)-1\right]$. Suppose $K \subset Z$ and $|K| \leq k$. Then, by the lemma, there exists $z \in\left[s, s+p_{k}(1)-1\right]$ such that $z \notin(-K+\bar{D})$. Thus $S_{k}+s \not \subset-K+A_{0}$ for all $s \in Z$, and $D$ is a $C^{\prime}$-subset of $Z$. 
A locally finite group is a group in which every finite subset generates a finite subgroup. Note that the countable locally finite groups are precisely those groups which can be obtained as an increasing union of finite subgroups.

4.5 Theorem. Let $G$ be an infinite group such that $G=\bigcup\left\{G_{i}: i \in N\right\}$ where each $G_{i}$ is a finite subgroup of $G$ and each $G_{i} \subset G_{i+1}$. Then if $A$ is a left thick subset of $G, A$ contains a $C$-subset $D$ of $G$. Moreover, if eacb $G_{i}$ is normal in $G$, then $A$ contains a $C^{\prime}$-subset of $G$.

Proof. We can assume that $\left[G_{i+1}: G_{i}\right] \geq 2^{i+1}$. Let $H_{1}=G_{1}$. There exists $d_{1,1} \in G$ such that $d_{1,1} \notin H_{1}$ and $H_{1} d_{1,1} \subset A$. Choose $G_{q}$ so that $H_{1} \cup H_{1} d_{1,1} \subset$ $G_{q}$ and let $H_{2}=G_{q}$. Suppose we have defined $H_{1}, \cdots, H_{n}$ and $d_{1,1}, \cdots, d_{n-1,1}$ where $n \geq 2$. There exists $d_{n, 1} \in G$ such that $d_{n, 1} \notin H_{n}$ and $H_{n} d_{n, 1} \subset A$. Choose $G_{p}$ such that $H_{n} \cup H_{n} d_{n, 1} \subset G_{p}$ and let $H_{n+1}=G_{p}$. In this way we get a sequence $\left(H_{i}\right)$ of subgroups of $G$ such that $G=\bigcup\left\{H_{i}: i \in N\right\}, H_{i} \cup H_{i} d_{i, 1} \subset H_{i+1}, d_{i, 1} k$ $H_{i}$, and $\left[H_{i+1}: H_{i}\right] \geq 2^{i+1}$. For each $i$ in $N$ let $d_{i, 0}=e$ and let $\left\{d_{i, 0}, d_{i, 1}, \cdots\right.$, $\left.d_{i, m(i)}\right\}$ be a right transversal of $H_{i}$ in $H_{i+1}$, where $d_{i, 1}$ is as above. If $i, j \in N$ and $j>i$, define

$$
T_{i, j}=\left\{d_{i, p(i)} d_{i+1, p(i+1)} \cdots d_{j-1, p(j-1)}: 0 \leq p(r) \leq m(r) \text { for } i \leq r \leq j-1\right\}
$$

and let $T_{i, i}=\{e\}$. Let $T_{i}=\bigcup\left\{T_{i, j}: j \geq i\right\}$. Then $T_{i, j}$ is a right transversal of $H_{i}$ in $H_{j}$ and $T_{i}$ is a right transversal of $H_{i}$ in $G$. Also, $T_{i, j} T_{j, k}=T_{i, k}$ if $i \leq j \leq$ $k$. If $1 \leq i<k$, define $B_{k, i}=\bigcup\left\{H_{i} d_{i, 1} t: t \in T_{i+1, k}\right\}$ and let $B_{k}=\bigcup\left\{B_{k_{0} i}: 1 \leq i<k\right\}$. Let $D=\bigcup\left\{\left(H_{k} \backslash B_{k}\right) d_{k, 1}: k \geq 2\right\}$.

For $j \in N$, define $\phi_{j} \in M(G)$ by $\phi_{j}(f)=\left|H_{j}\right|^{-1} \Sigma_{g \in H_{j}} f\left(g d_{j, 1}\right)$ for all $f \in m(G)$. Then if $s \in H_{j}$ and $f \in m(G)$ we have

$$
\phi_{j}(f)-\phi_{j}\left(l_{s} f\right)=\left|H_{j}\right|^{-1}\left(\sum_{g \in H_{j}} f\left(g d_{j, 1}\right)-\sum_{g \in H_{j}} f\left(s g d_{j, 1}\right)\right)=0,
$$

so the sequence $\left(\phi_{j}\right)$ is $w^{*}$ convergent to left invariance. Let $\phi$ be a $w^{*}$ limit point of $\left(\phi_{j}\right)$. If $n \geq 2$,

$$
\begin{aligned}
\left|B_{n}\right| & \leq \sum_{i=1}^{n-1}\left|B_{n, i}\right| \\
& =\sum_{i=1}^{n-1}\left|H_{i}\right|\left|T_{i+1, n}\right|=\sum_{i=1}^{n-1}\left|H_{i}\right|\left|H_{n}\right| /\left|H_{i+1}\right| \\
& \leq\left|H_{n}\right| \sum_{i=1}^{n-1} 2^{-(i+1)} \leq\left|H_{n}\right| / 2 .
\end{aligned}
$$

Hence, $\phi_{n}\left(\chi_{G \backslash D}\right)=\left|H_{n}\right|^{-1}\left|(G \backslash D) \cap H_{n} d_{n, 1}\right|=\left|H_{n}\right|^{-1}\left|B_{n}\right| \leq 1 / 2$. Thus $\bar{d}(D) \geq$ $\phi\left(\chi_{D}\right) \geq 1 / 2>0$. 
Now, let $K$ be a finite subset of $G$ and choose $m$ so that $K^{-1} \subset H_{m}$. We will show that no right translate of $H_{m+1}$ is contained in $H_{m} D$. We have $H_{m} D=$ $\bigcup\left\{H_{m}\left(H_{i} \backslash D_{i}\right) d_{i, 1}: i \geq 2\right\} \subset H_{m} \cup H_{m} d_{m, 1} \cup \bigcup\left\{\left(H_{i} \backslash H_{m} B_{i}\right) d_{i, 1}: i \geq m+1\right\}$. Suppose there exists $d \in T_{m+1}$ such that $H_{m+1} d \subset H_{m} D$. We have $H_{m} \cup H_{m} d_{m, 1} \subset$ $H_{m+1}$ and $H_{m+1} \cap \bigcup\left\{\left(H_{i} \backslash H_{m} B_{i}\right) d_{i, 1}: i \geq m+1\right\}=\varnothing$. We cannot have $H_{m+1} d C$ $H_{m} \cup H_{m} d_{m, 1}$ since $\left[H_{m+1}: H_{m}\right] \geq 2^{m+1}$. Hence we get $H_{m+1} d C$

$\bigcup\left\{\left(H_{i} \backslash H_{m} B_{i}\right) d_{i, 1}: i \geq m+1\right\}$. Since the $H_{i} d_{i, 1}$ 's are pairwise disjoint and $H_{m+1} \subset H_{i}$ for each $i \geq m+1$ this implies that there exists $r \geq m+1$ such that $H_{m+1} d \subset\left(H_{r} \backslash H_{m} B_{r}\right) d_{r, 1}$. Then we write $H_{m+1} d=H_{m+1} d^{\prime} d_{r, 1}$ where $d^{\prime} \in T_{m+1, r}$. We get $H_{m+1} d^{\prime} \cap H_{m} B_{r}=\varnothing$. But $H_{m} d_{m, 1} d^{\prime} \subset H_{m+1} d^{\prime}$ and $H_{m} d_{m, 1} d^{\prime} \subset B_{r}$ by the definition of $B_{r}$. Hence $H_{m} d_{m, 1} d^{\prime}=H_{m} H_{m} d_{m, 1} d^{\prime} \subset H_{m} B_{r}$, which is a contradiction. Therefore $H_{m} D$ is not left thick, so by Theorem $4.1, \bar{d}\left(K^{-1} D\right) \leq \bar{d}\left(H_{m} D\right)<1$.

We will use the following lemma to complete the proof.

4.6 Lemma. Under the bypotheses of Theorem 4.5, suppose each $G_{i}$ is normal in $G$ and let $k$ be in $N$. Then for each $j \in N$ there exists $q(k, j) \in N$ such that for each subset $K$ of $G$ with $|K| \leq k$ and for each right coset $H_{q(k, j)} g$ of $H_{q(k, j)}$ there is a coset $H_{j} g^{\prime}$ of $H_{j}$ with $H_{j} g^{\prime} \subset H_{q(k, j)} g$ and $H_{j} g^{\prime} \cap K^{-1} D=\varnothing$.

Proof. The proof is by induction on $k$. Let $q(1, j)=j+2$ for each $j \in N$. We will show first that $H_{j} d_{j, 1} t \cap D=\varnothing$ for all $t \in T_{j+1}, t \neq e$. Suppose there exists $t \in T_{j+1}$ such that $t \neq e$ and $H_{j} d_{j, 1} t \cap D \neq \varnothing$. Then there is an integer $i$ such that $H_{j} d_{j, 1} t \cap\left(H_{i} \backslash B_{i}\right) d_{i, 1} \neq \varnothing$. Since $H_{j} d_{j, 1} t \subset G \backslash H_{j+1}$ we must have $i \geq j+1$ and $H_{j} d_{j, 1} t \subset H_{i} d_{i, 1}$. There exists $t^{\prime} \in T_{j+1, i}$ such that $H_{j} d_{j, 1} t=H_{j} d_{j, 1} t^{\prime} d_{i, 1}$. But by the definition of $B_{i}, H_{j} d_{j, 1} t \subset B_{i}$. Hence $H_{j} d_{j, 1} t^{\prime} d_{i, 1} \cap\left(H_{i} \backslash B_{i}\right) d_{i, 1}=\varnothing$, which gives a contradiction and proves our assertion.

Let $g$ be a fixed element of $G$. Then $g^{-1} H_{j} d_{j, 1} t \cap g^{-1} D=\emptyset$ for all $t \in T_{j+1}$, $t \neq e$. Let $H_{q(1, j)^{d}}$ be any coset of $H_{q(1, j)^{\circ}}$ Choose $d^{\prime} \in T_{q(1, j)}$ such that

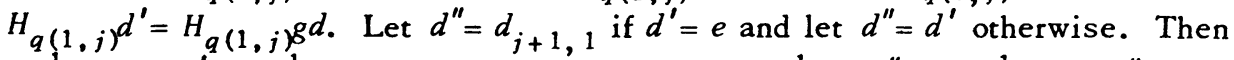
$g^{-1} H_{q(1, j)} d^{\prime}=g^{-1} H_{q(1, j)} g^{d}=H_{q(1, j)}$, and $H_{j}\left(g^{-1} d_{j, 1} d^{\prime \prime}\right)=g^{-1} H_{j} d_{j, 1} d^{\prime \prime} C$ $g^{-1} H_{j+1} d^{\prime \prime} \subset g^{-1} H_{q(1, j)} d^{\prime}=H_{q(1, j)} d$. Since $d^{\prime \prime} \in T_{j+1}$, we get $H_{j}\left(g^{-1} d_{j, 1} d^{\prime \prime}\right) \cap$ $g^{-1} D=g^{-1} H_{j} d_{j, 1} d^{\prime \prime} \cap g^{-1} D=\varnothing$, which proves the lemma for $k=1$.

Suppose the lemma holds for $k=n$. Let $q(n+1, j)=q(n, q(1, j))$ for each $j \in N$. Assume $K \subset G$ and $1 \leq|K| \leq n+1$. We can write $K=J \cup\{s\}$ where $1 \leq$

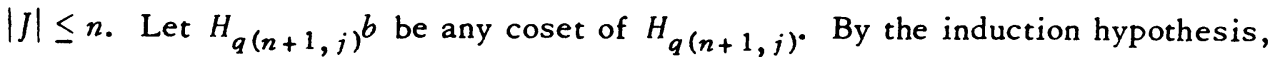
$H_{q(n+1, j)^{b}}=H_{q(n, q(1, j))^{b}}$ contains a coset $H_{q(1, j)} b^{\prime}$ of $H_{q(1, j)}$ such that $H_{q(1, j)^{b}} b^{\prime}$ $J^{-1} D=\varnothing$. Also $H_{q(1, j)} b^{\prime}$ contains a coset $H_{j} b^{\prime \prime}$ of $H_{j}$ such that $H_{j} b^{\prime \prime} \cap s^{-1} D=\varnothing$. Therefore, $H_{j} b^{\prime \prime} \cap K^{-1} D=\varnothing$ and $H_{j} b^{\prime \prime} \subset H_{q(1, j)^{b}} b^{\prime} \subset H_{q(n+1, j)^{b}}$, which proves the lemma.

To complete the proof of Theorem 4.5, assume each $G_{i}$ is normal in $G$ and let $S_{k}=H_{q(k, 1)}$ for each $k \in N$. Suppose $K \subset G$ and $|K| \leq k$. If $s \in G$, then $S_{k} s=$ $H_{q(k, 1)} s$ contains a coset $H_{1} d$ of $H_{1}$ such that $H_{1} d \cap K^{-1} D=\emptyset$. Thus $S_{k} s \not \subset$ $K^{-1} D$ for all $s \in G$. Therefore $D$ is a $C^{\prime}$-subset of $G$. 
Suppose that $G$ is a countably infinite left amenable group and that every left thick subset of $G$ contains a $C$-subset. Then if $A$ is any left thick subset of $G$, there is a set $\mathfrak{A}$ consisting of $C$-subsets of $G$ which are contained in $A$, such that $|\mathfrak{A}|=c$ and $D \cap E$ is finite for all $D, E \in \mathscr{U}$ with $D \neq D^{\prime}$. (See Chou [1, p. 780]. The argument there can be generalized.) Hence if $D, E \in \mathfrak{U}$ and $D \neq E$, we have $\left(D^{-} \backslash D\right) \cap\left(E^{-\backslash} \backslash E\right)=\varnothing$. Since supp $P \mu \subset \beta G \backslash G$ for each $\mu \in M l(G)$, we have shown that for each left thick subset $A$ of $G$ there is a set $M_{A}$ of extreme left invariant means on $G$ such that $\left|M_{A}\right|=c$, supp $P \mu \cap$ supp $P \nu=\emptyset$ for $\mu, \nu \in M_{A}$ with $\mu \neq \nu$, and supp $P \mu$ is not a minimal set if $\mu \in M_{A}$.

4.7 Theorem. Suppose $G$ is an abelian group and suppose a subgroup $H$ of $G$ bas a $C^{\prime}$-subset $B^{\prime}($ of $H)$. Then there exists a $C^{\prime}$-subset of $G$.

Proof. Let $T$ be a transversal of $H$ in $G$ such that $e \in T$ and let $B=T B^{\prime}$. Let $\phi$ be a left invariant mean on $m(H)$ such that $\phi\left(\chi_{B}\right)>0$ and let $\mu$ be any left invariant mean on $m(G)$. If $f \in m(G)$, define $P f \in m(G)$ by $P f(g)=\phi\left(\left.l_{g} f\right|_{H}\right)$ for all $g \in G$. Define $\nu \in M(G)$ by $\nu(f)=\mu(P f)$ for all $f \in m(G)$. Then $\nu \in M l(G)$. If $b \in H$ and $t \in T$ we have $l_{t} \chi_{B}(b)=\chi_{B}(t b)=\chi_{B^{\prime}}(b)$, so $\left.\left(l_{t} \chi_{B}\right)\right|_{H}=\chi_{B^{\prime}}$. Hence $P \chi_{B}(t b)=$ $\phi\left(\left.l_{t b} \chi_{B}\right|_{H}\right)=\phi\left(\left.l_{b} \chi_{B}\right|_{H}\right)=\phi\left(\chi_{B^{\prime}}\right)$. Thus $\bar{d}(B) \geq \nu\left(\chi_{B}\right)=\mu\left(P \chi_{B}\right) \geq \phi\left(\chi_{B^{\prime}}\right)>0$.

Suppose $k \in N$ and let $S_{k}$ be a subset of $H$ with the property that $S_{k} b \not \subset J^{-1} B^{\prime}$ for any $b$ in $H$ and for any subset $J$ of $H$ with $|J| \leq k^{2}$. Suppose $K \subset G$ and $|K| \leq$ $k$. We can write $K^{-1}=\bigcup\left\{t_{i} K_{i}: 1 \leq i \leq n\right\}$ where each $K_{i} \subset H, n \leq k$, and $t_{1}, \cdots$, $t_{n} \in T$. Let $K^{\prime}=\bigcup\left\{K_{i}: 1 \leq i \leq n\right\}$ and let $K_{0}=\bigcup\left\{t_{i} K^{\prime}: 1 \leq i \leq n\right\}$. Then. $K^{-1} \mathrm{C}$ $K_{0},\left|K^{\prime}\right| \leq k$, and $\left|K_{0}\right| \leq k^{2}$. We will show that $S_{k} s \not \subset K_{0} B$ for all $s \in G$. We have $K_{0} B=\bigcup\left\{t_{i} K^{\prime} T B^{\prime}\right\}=\bigcup\left\{t_{i} K^{\prime} t B^{\prime}: 1 \leq i \leq n, t \in T\right\}$. For each $i \leq n$ and $t \in T$ let $g_{i, t}$ be the unique element of $T$ such that $t_{i} K^{\prime} t \subset g_{i, t} H$ and let $K_{i, t}=g_{i, t}^{-1} t_{i} K^{\prime} t \subset$ $H$. Then, for fixed $i$, the set $\left\{g_{i, t}: t \in T\right\}$ is a transversal for $H$ in $G$. If $d \in T$, let $K_{d}=\bigcup\left\{K_{i, t}: g_{i, t}=d\right\}$. Note that $\left|K_{i, t}\right|=\left|K^{\prime}\right| \leq k$, so $\left|K_{d}\right| \leq n\left|K^{\prime}\right| \leq k^{2}$. We have $K_{0} B=\bigcup\left\{g_{i, t} K_{i, t} B^{\prime}: 1 \leq i \leq n, t \in T\right\}=\bigcup\left\{d K_{d} B^{\prime}: d \in T\right\}$. Also, $s_{k} t H C$ $H t b=t H$ for all $t \in T$ and $b \in H$. Suppose $S_{k} t b \subset K_{0} B$ for some $t \in T$ and $b \in H$. Then we would have $t S_{k} b=S_{k} t b \subset K_{0} B \cap t H=t K_{t} B^{\prime}$. This gives $S_{k} b \subset K_{t} B_{0}$, which contradicts the definition of $S_{k}$. Hence $S_{k} g \not \subset K_{0} B \supset K^{-1} B$ for all $g \in G$, so $B$ is a $C^{\prime}$ subset of $G$.

4.8 Corollary. If $G$ is an infinite abelian group then $G$ bas a $C^{\prime}$-subset and there is an extreme left invariant mean $\phi$ sucb that $\phi$ is not in the $w^{*}$ closed convex bull of the set $\{\mu \in M l(G)$ : supp $P \mu$ is a minimal set $\}$.

Proof. $G$ has either a subgroup which is isomorphic to $Z$ or a countable periodic subgroup. Thus, by 4.3 and $4.5, G$ has a subgroup with a $C^{\prime}$-subset. By 4.7, $G$ has a $C^{\prime}$-subset and Corollary 3.12 gives the result.

The next theorem can be proved by methods similar to those used in the proof of Theorem 4.7. We omit the proof. 
4.9 Theorem. If $S$ is an infinite abelian cancellation semigroup, then $S$ bas a $C^{\prime}$-subset.

4.10 Proposition. Let $G$ be a left amenable group with a normal subgroup $H$. Suppose $G / H$ contains a $C$-subset $\left[C^{\prime}\right.$-subset $B$. Then $G$ contains a $C$-subset [C'subset].

Proof. Let $T$ be a transversal of $H$ in $G$ and let $T_{0}=\{t \in T: t H \in B\}$. Let $\phi$ be a left invariant mean on $m(G / H)$ such that $\phi\left(\chi_{B}\right)>0$ and let $\psi$ be any left invariant mean on $m(H)$. If $f \in m(G), t \in T$, and $b \in H$, then $\psi\left(\left.l_{t b} f\right|_{H}\right)=\psi\left(\left.l_{b} l_{t} f\right|_{H}\right)=$ $\psi\left(l_{t} f l_{H}\right)$. If $f \in m(G)$, define $P f \in m(G / H)$ by $P f(t H)=\psi\left(\left.l_{t} f\right|_{H}\right)$; then $P f$ does not depend on $T$. If we let $\mu(f)=\phi(P f)$ for all $f \in m(G)$, then $\mu$ is a left invariant mean on $m(G)$. Let $A=T_{0} H \subset G$. If $t H \in B$, then $t H \subset A$ so $H \subset t^{-1} A$ and $l_{t} \chi_{A}(b)=$ $\chi_{t-1}(b)=1$ for all $b \in H$. Thus $P \chi_{A}(t H)=1$. If $t H \in B$ then $A \cap t H=\emptyset$, so $t^{-1} A \cap H=\varnothing$ and $l_{t} \chi_{A}(b)=0$ for all $b \in H$. This gives $P \chi_{A}(t H)=0$. Thus, $P \chi_{A}=$ $\chi_{B}$ and $\bar{d}(A) \geq \mu\left(\chi_{A}\right)=\phi\left(\chi_{B}\right)>0$.

Let $K$ be a finite subset of $G$ and let $i$ be the canonical map of $G$ onto $G / H$. Let $J \subset G / H$ be a finite set such that $J y \not \subset i(K)^{-1} B$ for all $y \in G / H$. Let $V=$ $\{t \in T: t H \in J\}$. Suppose there exists $g \in G$ such that $V g \subset K^{-1} A$. Then we would have $J i(g)=i(V g) \subset i\left(K^{-1} A\right)=i(K)^{-1} B$ which is impossible by the choice of $J$. Hence $A$ is a $C$-subset of $G$.

Now assume $B$ is a $C^{\prime}$-subset of $G / H$. If $k \in N$, let $S_{k}$ be a finite subset of $G / H$ such that $S_{k} y \subset K^{-1} B$ for all $K \subset G / H$ with $|K| \leq k$ and for all $y \in G / H$. Let $V_{k}=$ $\left\{t \in T: t H \in S_{k}\right\}$. Then if $J \subset G,|J| \leq k$, and $g \in G$, the assertion $V_{k} g \subset J^{-1} A$ would imply that $S_{k} i(g) \subset i(J)^{-1} B$, which contradicts the definition of $S_{k}$. Therefore $A$ is a $C^{\prime}$-subset of $G$.

4.11 Proposition. Let $G$ be a left amenable group and let $H$ be a subgroup of finite index in G. Suppose $A$ is a C-subset [C'-subset] of H. Then $A$ is a C-subset $\left[C^{\prime}\right.$-subset $]$ of $G$.

Proof. Let $\phi$ be a left invariant mean on $m(H)$ such that $\phi\left(\chi_{A}\right)>0$. If $f \in m(G)$, define $P f \in m(G)$ by $P f(g)=\phi\left(\left.l_{g} f\right|_{H}\right)$ for all $g \in G$. Choose $\psi \in M l(G)$ and define $\mu \in M(G)$ by $\mu(f)=\psi(P f)$ for all $f \in m(G)$. Then $\mu \in M l(G)$. If $b \in H$, then $P \chi_{A}(b)=$ $\phi\left(\left.l_{b} \chi_{A}\right|_{H}\right)=\phi\left(\chi_{A}\right)$. If $g \in G \backslash H$, then $P \chi_{A}(g)=\phi\left(\left.l_{g} \chi_{A}\right|_{H}\right)=\phi\left(\chi_{g}-1_{A \cap H}\right)=0$. Thus $P \chi_{A}=\phi\left(\chi_{A}\right) \chi_{H}$. Also, $\mu\left(\chi_{H}\right)=[G: H]^{-1}$. Thus $\bar{d}(A) \geq \mu\left(\chi_{A}\right)=\phi\left(\chi_{A}\right) \mu\left(\chi_{H}\right)$ $>0$. It is clear that $A$ satisfies the second condition for a $C$-subset [ $C^{\prime}$-subset] of $G$.

4.12 Theorem. Every infinite solvable group $G$ contains a $C^{\prime}$-subset.

Proof. Let $H_{0}=\{e\}, H_{1}, \cdots, H_{n}=G$ be a normal series for $G$ such that $H_{i} / H_{i-1}$ is abelian for $1 \leq i \leq n$. Since $G$ is infinite we can define $m=$ $\sup \left\{i: H_{i} / H_{i-1}\right.$ is infinite $\}$. Then by $4.8, H_{m} / H_{m-1}$ has a $C^{\prime}$-subset. Thus, by 
4.10, $H_{m}$ contains a $C^{\prime}$-subset. If $H_{m}=G$ we are done. Otherwise, for $0 \leq p \leq$ $n-m-1, H_{m+p}$ contains a $C^{\prime}$-subset implies, by 4.11 , that $H_{m+p+1}$ contains a $C^{\prime}$-subset. By induction, $H_{n}=G$ contains a $C^{\prime}$-subset.

We do not know whether there are any left amenable semigroups $S$, aside from those which have finite invariant subsets in $\beta S$ (see [5]), which do not have $C$ subsets.

\section{REFERENCES}

1. C. Chou, Minimal sets and ergodic measures for $\beta N \backslash N$, Illinois J. Math. 13 (1969), 777-788. MR 40 \#2814.

2. M. M. Day, Amenable semigroups, Illinois J. Math. 1 (1957), 509-544. MR 19, 1067.

3. L. Gillman and M. Jerison, Rings of continuous functions, University Series in Higher Math., Van Nostrand, Princeton, N. J., 1960. MR 22 \#6994.

4. W. H. Gottschalk and G. A. Hedlund, Topological dynamics, Amer. Math. Soc. Colloq. Publ., vol. 36, Amer. Math. Soc., Providence, R. I., 1955. MR 17, 650.

5. A. Lau, Topological semigroups with invariant means in the convex hull of the multiplicative means, Trans. Amer. Math. Soc. 148 (1970), 69-84. MR 41 \#1911.

6. T. Mitchell, Constant functions and left invariant means on semigroups, Trans. Amer. Math. Soc. 119 (1965), 244-261. MR 33 \#1743.

7. R. R. Phelps, Lectures on Choquet's theorem, Van Nostrand, Princeton, N. J., 1966. MR $33 \# 1690$.

8. C. Wilde, On amenable semigroups and applications of the Stone-Čech compactification, Thesis, University of Illinois, Urbana, Ill., 1964.

9. C. Wilde and K. Witz, Invariant means and the Stone-ČCech compactification, Pacific J. Math 21 (1967), 577-586. MR 35 \#3423.

DEPARTMENT OF MATHEMATICS, SUNY, COLLEGE AT PLATTSBURGH, PLATTSBURGH, NEW YORK 12901 